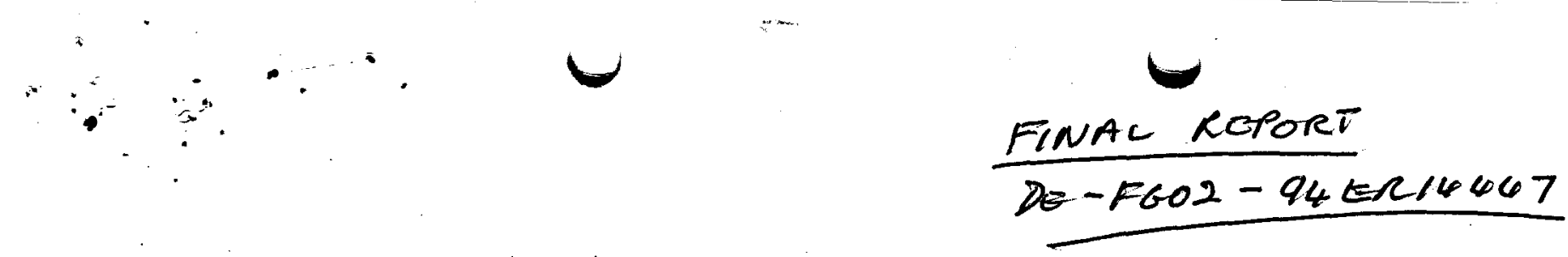

\title{
SYNTHESIS AND OPTIMIZATION OF INTEGRATED CHEMICAL PROCESSES
}

Paul I. Barton

Department of Chemical Engineering

Massachusetts Institute of Technology

617-253-6526

FAX: 617-258-5042

Email: pib@mit.edu

Lawrence B. Evans

Department of Chemical Engineering

Massachusetts Institute of Technology

617-577-0100 x310

FAX: 617-577-0722

Email: evans@aspentech.com

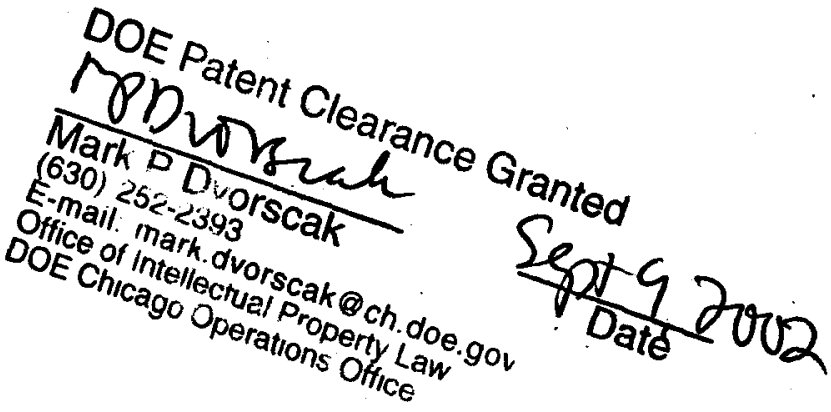

\section{Optimal Batch Process Development}

During the course of this project we have completed the following tasks:

1. completed the initial work on screening models for batch process development, which have appeared as a two part article $[2,1]$.

2. undertaken a detailed study of the 'novel' middle vessel batch distillation configuration when applied to the separation of homogeneous azeotropic mixtures $[4,5,6]$.

3. the first steps in extending the theory of azeotropic batch distillation to heterogeneous mixtures (i.e.,

- those in which two or more liquid phases may form in equilibrium with the vapor phase) has led to a novel theory and algorithm for the computation of all the heterogeneous azeotropes present in an arbitrary multicomponent mixture $[12,13]$.

The work in the latter two categories is described in more detail in the following subsections.

\section{Azeotropic Distillation in a Middle Vessel Batch Column}

The Middle Vessel Batch Column (MVC) is a (relatively) novel distillation configuration that has attracted much academic attention in recent years. We were interested in the possibility of using the MVC as a flexible solvent recovery technology to break azeotropic mixtures, particularly in flexible manufacturing systems such as pharmaceutical processes. This enables solvent and unreacted reagents to be recovered from waste streams and recycled, rather than being treated as toxic waste.

A mathematical model for the MVC was developed that was suitable for studying its qualitative dynamics when applied to the separation of multicomponent azeotropic mixtures. A limiting analysis was developed with this model that elucidated the separation sequences that can be achieved with the MVC, and how these separation sequences vary with the key operating parameter, the relative rate at which top and bottom products are drawn from the column. This enables a complete characterization of the MVC dynamics in the presence of linear separation boundaries.

We then considered the more realistic case in which the separation boundaries are nonlinear. The differences between these boundaries in the two extreme cases of operation as a batch stripper or as a batch rectifier results in the MVC pot composition being able to cross separation boundaries. On the basis of these insights, operating policies were developed in which azeotropic mixtures can be separated into their constituent pure components in a single multipurpose MVC. These operating policies were also shown to 


\section{DISCLAIMER}

This report was prepared as an account of work sponsored by an agency of the United States Government. Neither the United States Government nor any ageney thereof, nor any of their employees, makes any warranty, express or implied, or assumes any legal liability or responsibility for the accuracy, completeress, or usefulness of any information, apparatus, product, or process diselosed, or represents that its use would not infringe privately owned rights. Reference herein to any specific commercial product, process, or service by trade name, trademark, manufacturer, or otherwise does not necessarily constitute or imply its endorsement, recommendation, or favoring by the United States Government or any agency thereof. The views and opinions of authors expressed herein do not necessarily state or reflect those of the United States Governmeat or any agency thereof. 


\section{DISCLAIMER}

Portions of this document may be illegible in electronic image products. Images are produced from the best available original document. 


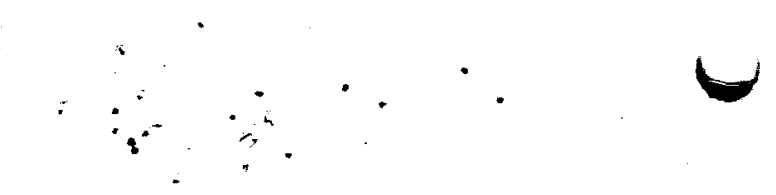

be the time analogues of sequences of continuous distillation columns that achieve the same azeotropic separation. Based on this, we conjecture that many complex separations currently only achieved with dedicated sequences of continuous distillation columns can also be reproduced as sequential operating policies over time in a single MVC. Thus, the MVC represents the ultimate multipurpose solvent recovery technology for flexible manufacturing systems because it can handle, in a batch multipurpose mode, separations that would otherwise require dedicated continuous distillation sequences. Finally, the characteristics of perfect MVC entrainers which allow the complete separation of any azeotropic into its constituent pure components were elucidated. All these theoretical developments were validated using detailed dynamic models of the MVC, using the robust numerical technologies previously developed under this contract.

\section{Computation of Heteroazeotropes}

A key component of the screening model approach to batch process development is the use of the fixed points (pure components and azeotropes) of a simple distillation residue curve map to characterize the alternative separation sequences possible in the process structure to be synthesized. Many industrial processes involve heterogeneous azeotropic mixtures. It is therefore necessary to extend the detailed qualitative understanding that now exists for batch distillation of homogeneous azeotropic mixtures to their heterogeneous counterpart.

As the first step, we have developed a novel and efficient algorithm that computes all the simple distillation fixed points of an arbitrary multicomponent liquid mixed (pure components, homogeneous azeotropes and heterogeneous azeotropes). Our approach is an extension of the homotopy continuation approach for homogeneous mixtures developed by Fidkowski and co-workers [7]. The new approach is independent, of the representation of thermodynamic nonideality, the topology of the liquid-liquid region, and can handle any number of liquid (and even solid) phases in equilibrium. Much of the efficiency of our approach is derived from minimizing the number of extremely expensive phase stability calculations that need to be performed. In addition, we have developed an extensive theory that establishes very mild conditions under which it is guaranteed that all homogeneous and heterogeneous azeotropes present in the mixture will be computed by our algorithm.

We have also extended our approach to compute efficiently changes in phase equilibrium structure with parameters such as pressure, such as the appearance and/or disappearance of azeotropes and heteroazeotropes. This enables one to detect incipient azeotropes that may exist under different conditions, thus elucidating more separation possibilities or separation difficulties if the process conditions are altered.

\section{Plant Wide Dynamic Optimization}

During the course of this project we have completed the following tasks:

1. developed a complete theory (including existence and uniqueness theorems) for the parametric sensitivities of hybrid discrete/continuous dynamic systems [9].

2. conducted and published the pioneering work on the numerical optimal control of hybrid discrete/continuous systems $[8,3]$.

3. explored three novel approaches to the treatment of path inequality constraints in numerical optimal control.

The work in these categories is described in more detail in the following subsections.

\section{Parametric Sensitivity Analysis of Hybrid Discrete/Continuous Systems}

Many engineering applications call for the analysis of a discontinuous (or hybrid discrete/continuous) dynamic systems. Examples include the design of operating procedures for process start-up, shut-down and changeovers, the design of emergency shut-down systems, or the optimal design of inherently dynamic processes such as those operated in a batch, semi-continuous and/or periodic manner.

In this task, we investigated novel extensions of the classical parametric sensitivity analysis results for (continuous) ODEs to a quite general class of hybrid discrete/continuous systems with ODEs or DAEs embedded. The first step was to derive general formulas describing the transfer of the parametric sensitivity 


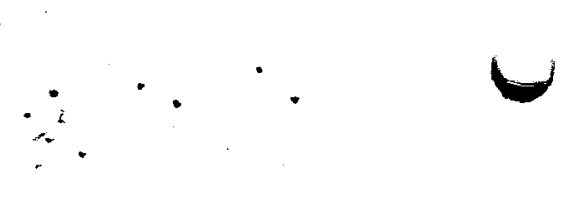

values at events (discontinuities) along the state trajectory. These results show that the sensitivities exhibit a characteristic 'jump' in their values in response to a discontinuity in the state trajectories. In addition, they enable an efficient extension of the numerical sensitivity analysis algorithms previously developed under this proposal for the computation of hybrid sensitivity trajectories.

The second step was to establish the key existence and uniqueness results for hybrid sensitivity trajectories. These novel theorems establish sufficient conditions for existence and uniqueness of the sensitivities for hybrid systems embedded with nonlinear ODEs and linear time invariant DAEs. In particular, these results show that the sensitivities of a hybrid system do indeed exist almost everywhere in parameter space. In addition, there are certain 'critical' parameter values at which the conditions of the theorems do not hold, and these values are most often associated with qualitative changes in the sequence of events that occur along the state trajectory.

\section{Numerical Optimal Control of Hybrid Discrete/Continuous Systems}

In this task we conducted the pioneering research on numerical optimal control of hybrid discrete/continuous dynamic systems, our primary motivation being the development of an optimization technology that can automatically design operating procedures for chemical processes using detailed dynamic models. The most intriguing class of problems are those in which the optimal trajectories are characterized by a sequence of switches and/or jumps at events, some of which are dependent on the state of the system satisfying certain conditions (state or implicit events), and it is necessary to search over several alternative sequences of events to find the optimal operating procedure.

The potential of numerical optimization procedures to make optimal sequencing decisions in hybrid dynamic systems was explored. A general formulation of the hybrid optimal control problem was developed. However, the classical optimality conditions for such problems provide no guidance on how to find the optimal sequence. Instead, we reformulated the problem as a parameter optimization problem via discretization of the control profiles (control parameterization). An extension of the results concerning hybrid sensitivities then proved the novel theoretical result that parameter optimization of hybrid dynamic systems (including sequencing decisions) is in general a nonsmooth problem, but also smooth in many important cases. For illustration, the design of a minimum time changeover operation for a pressure vessel avoiding the formation of explosive mixtures was considered. Results show that a direct stochastic search (which is relatively insensitive to nonsmoothness in the optimization formulation) procedure can indeed make the sequencing decisions automatically given merely a statement of the system model and the path and point constraints.

We have also prepared an invited review article on the state-of-the-art in modeling, simulation, sensitivity analysis and optimization of hybrid systems [11].

\section{Optimal Control with Path Inequality Constraints}

We developed three novel approaches to the treatment of inequality path constraints in numerical optimal. control: a) a reformulation of a classical approach [10] in light of modern understanding of high-index DAEs, b) a reformulation of the problem as a hybrid discrete/continuous dynamic optimization, and c) reformulation of the problem to avoid nonsmoothness in the resulting optimization problem. The insights gained through a) served to elucidate the fundamental limitations of that line of approach to the problem. Similarly, approach b) is limited by the fact that if the sequence of constraint activations/deactivations along the state trajectory can change depending on the position in parameter space, then the optimization problem will be nonsmooth. These conclusions inspired approach c) in which the sequence of constraint activations/deactivations is fixed, and then the optimal trajectories are found for this sequence. This yielded a highly efficient approach that exhibited impressive speed and performance on all the standard literature problems, and a large-scale process start-up problem that we developed. However, this approach requires a higher level decision process (basically a mixed-integer dynamic optimization) in order to search over alternative sequences. It is concluded that further progress on path inequality constrained problems will come from progress on generic approaches to numerical optimal control of hybrid systems. 


\section{Recent Publications from this Research (Since Sept. 1997)}

\section{Refereed Publications}

1. Feehery W. F., J. E. Tolsma and P. I. Barton, "Efficient Sensitivity Analysis of Large-Scale DifferentialAlgebraic Systems", Applied Numerical Mathematics, 25(1):41-54, 1997.

2. Barton P. I., R. J. Allgor, W. F. Feehery, and S. Galán, "Dynamic Optimization in a Discontinuous World", Ind. Eng. Chem. Res., 37(3):966-981, 1998.

3. Tolsma J. E. and P. I. Barton, "On Computational Derivatives", Computers chem. Engng., 22(4/5):475490, 1998.

4. Galán S. and P. I. Barton, "Dynamic Optimization of Hybrid Systems", Computers chem. Engng., 22(S):S183-S190, 1998.

5. Tolsma J. E. and P. I. Barton, "Computation of Heteroazeotropes", Computers chem. Engng., 22(S):S61$\mathrm{S} 68,1998$.

6. Feehery W. F. and P. I. Barton, "Dynamic Optimization with State Variable Path Constraints", Computers chem. Engng., 22(9):1241-1256, 1998.

7. Cheong W. and P. I. Barton, "Azeotropic Distillation in a Middle Vessel Batch Column. 1. Model Formulation and Linear Separation Boundaries", Ind. Eng. Chem. Res., 38(4):1504-1530, 1999.

8. Cheong W. and P. I. Barton, "Azeotropic Distillation in a Middle Vessel Batch Column. 2. Nonlinear Separation Boundaries", Ind. Eng. Chem. Res., 38(4):1531-1548, 1999.

9. Cheong W. and P. I. Barton, "Azeotropic Distillation in a Middle Vessel Batch Column. 3. Model Validation", Ind. Eng. Chem. Res., 38(4):1549-1564, 1999.

10. Allgor R. J. and P. I. Barton, "Mixed Integer Dynamic Optimization I: Problem Formulation", Computers chem. Engng., 23(4/5):567-584, 1999.

11. Feehery W. F. and P. I. Barton, "Dynamic Optimization with Equality Path Constraints", Ind. Eng. Chem. Res., 38(6):2350-2363, 1999.

12. Galán S., W. F. Feehery and P. I. Barton, "Parametric Sensitivity Functions for Hybrid Discrete/Continuous Systems", Applied Numerical Mathematics, 31(1):17-48, 1999.

13. Tolsma J. E. and P. I. Barton, "Efficient Calculation of Sparse Jacobians", SIAM Journal on Scientific Computing, 20(6):2282-2296, 1999.

14. Allgor R. J., L. B. Evans and P. I. Barton, "Screening Models for Batch Process Development. I. Design Targets for Reaction/Distillation Networks", Chemical Engineering Science, 54(19):4145-4164, 1999.

15. Allgor R. J., and P. I. Barton, "Screening Models for Batch Process Development. II. Case Studies", Chemical Engineering Science, 54(18):4065-4087, 1999.

16. Tolsma J. E. and P. I. Barton, "Computation of Heteroazeotropes. I: Theory", Chemical Engineering Science, 55(18):3817-3834, 2000.

17. Tolsma J. E. and P. I. Barton, "Computation of Heteroazeotropes. II: Efficient Calculation of Changes in Phase Equilibrium Structure", Chemical Engineering Science, 55(18):3835-3853, 2000.

18. Barton P. I., J. R. Banga and S. Galán, "Optimization of Hybrid Discrete/Continuous Dynamic Systems", Computers and Chemical Engineering, 24(9/10):2171-2182, 2000.

19. Barton P. I., "Solvent Recovery Opportunities in the Pharmaceutical Industry", Current Opinion in Drug Discovery \& Development, 3(6):707-713, 2000.

20. Cheong W. and P. I. Barton, "Pollution Prevention with a Middle Vessel Column", In: Process Design Tools for the Environment, M. El-Halwagi and S. Sikdar, editors, 219-245, Taylor and Francis, 2001.

21. Barton P. I. and C. K. Lee, "Modeling, Simulation, Sensitivity Analysis and Optimization of Hybrid Systems", Submitted to: ACM Transactions on Modeling and Computer Simulation, September 2001. 


\section{Theses}

1. Allgor, R. J., "Modeling and Computational Issues in the Development of Batch Processes", Ph.D. Thesis, MTT, June 1997.

2. Fèehery, W. F., "Dynamic Optimization with Path Constraints", Ph.D. Thesis, MIT, March 1998.

3. Cheong, W. Y., "Simulation and Analysis of a Middle Vessel Batch Distillation Column", B.Sc. Thesis, MIT, May 1998.

4. Tolsma, J. E., "Analysis of Heteroazeotropic Systems", Ph.D. Thesis, MIT, April 1999.

\section{Keynote, Plenary and Invited Papers}

1. Barton P. I., "Dynamic Optimization in a Discontinuous World", Aspen World, 11-15th October 1997, Boston, USA.

2. Barton P. I., "Modeling, Simulation and Optimization of Discrete/Continuous Dynamic Systems", In: Christina Skjöldebrand and Gilles Trystram, editors, Automatic Control of Food and Biological Processes IV, pp. 125-145, 21st-23rd September 1998, Göteburg, Sweden.

3. Barton P. I., B. S. Ahmad, W. Cheong and J. E. Tolsma, "Synthesis of Batch Processes with Integrated Solvent Recovery", In: S. K. Sikdar and U. Diwekar, editors, Tools and Methods for Pollution Prevention, pp. 205-231, 12-14th October 1998, Prague, Czech Republic, NATO Science Series, 2/62, Kluwer, 1999.

4. Barton P. I., "Designing Flexible Manufacturing Systems with Integrated Solvent Recovery", NSF Workshop on Hybrid Technologies for Waste Minimization, Breckenridge, Colorado, 15-16th July, 1999.

5. Barton P. I., "Optimization of Hybrid Discrete/Continuous Dynamic Systems", II Pan American Workshop on Catalysis \& Process Systems Engineering, Santa Fe, Argentina, 2nd-3rd September, 1999.

6. Barton P. I., "Sensitivity Analysis of Hybrid Systems: Theory and Numerical Implications", Sixth Copper Mountain Conference on Iterative Methods, Copper Mountain, Colorado, 3rd-7th April, 2000.

7. Barton P. I., "Modeling, Simulation and Sensitivity Analysis of Hybrid Systems", In: L. K. Mestha, editor, Proceedings of IEEE International Symposium on Computer Aided Control System Design, Anchorage, Alaska, 25-27th September 2000.

8. Barton P. I., "Hybrid Systems: A Powerful Framework for the Analysis of Process Operations", In: 1. Hashimoto, editor, Proceedings PSE Asia 2000: International Symposium on Design, Operation and Control of Next Generation Chemical Plants, 6-8th December 2000, Kyoto, Japan.

9. Barton P. I. and J. E. Tolsma, "Hidden Discontinuities and Parametric Sensitivity Calculations", Sensitivity Analysis Workshop 2001, Lawrence Livermore National Laboratory, Livermore, California, 16-17th August 2001.

\section{Presented Papers and Conference Proceedings}

1. Galán S. and P. I. Barton, "Dynamic Optimization Formulations for Operating Procedure Synthesis", AIChE Annual Meeting, Los Angeles, 16th-21st November, 1997.

2. Barton P. I., J. R. Banga and S. Galán, "Optimization of Discontinuous Dynamic Systems", Proceedings of the Sixteenth Symposium on Energy Engineering Sciences, 13-15th May 1998, Argonne, Illinois.

3. Galán S. and P. I. Barton, "Dynamic Optimization of Hybrid Systems", ESCAPE-8, Brugge, Belgium, May 24-27th 1998.

4. Tolsma J. E. and P. I. Barton, "Computation of Heteroazeotropes", ESCAPE-8, Brugge, Belgium, May 24-27th 1998.

5. Cheong W. and P. I. Barton, "Distillation of Azeotropic Mixtures in a Middle Vessel Batch Column", AIChE Annual Meeting, Miami Beach, November 1998. 
6. Galán S., J. R. Banga and P. I. Barton, "Automatic Generation of Operating Procedures", AIChE Annual Meeting, Miami Beach, November 1998.

7. Tolsma J. E. and P. I. Barton, "Analysis of Heteroazeotropic Systems", AIChE Annual Meeting, Miami Beach, November 1998.

\section{References}

[1] R. J. Allgor and P. I. Barton. Screening models for batch process development: II. Case studies. Chemical Engineering Science, 54(18):4065-4087, 1999.

[2] R. J. Allgor, L. B. Evans, and P. I. Barton. Screening models for batch process development: I. Design targets for reaction/distillation networks. Chemical Engineering Science, 54(19):4145-4164, 1999.

[3] P. I. Barton, J. R. Banga, and S. Galán. Optimization of hybrid discrete/continuous dynamic systems. Computers chem. Engng, 4(9/10):2171-2182, 2000.

[4] W. Cheong and P. I. Barton. Azeotropic distillation in a middle vessel batch column: 1. Model formulation and linear separation boundaries. Industrial \& Engineering Chemistry Research, 38(4):1504-1530, 1999.

[5] W. Cheong and P. I. Barton. Azeotropic distillation in a middle vessel batch column: 2. Nonlinear separation boundaries. Industrial \& Engineering Chemistry Research, 38(4):1531-1548, 1999.

[6] W. Cheong and P. I. Barton. Azeotropic distillation in a middle vessel batch column: 3. Model validation. Industrial \& Engineering Chemistry Research, 38(4):1549-1564, 1999.

[7] Z. T. Fidkowski, M. F. Malone, and M. F. Doherty. Computing azeotropes in multicomponent mixtures. Computers chem. Engng, 17:1141, 1993.

[8] S. Galán and P. I. Barton. Dynamic optimization of hybrid systems. Computers chem. Engng, 22(S):S183-S190, 1998.

[9] S. Galán, W. F. Feehery, and P. I. Barton. Parametric sensitivity functions for hybrid discrete/continuous systems. Applied Numerical Mathematics, 31(1):17-48, 1999.

[10] D. H. Jacobson and M. M. Lele. A transformation technique for optimal control problems with a state variable inequality constraint. IEEE Trans. Automatic Control, 5:457-464, 1969.

[11] C. K. Lee and P. I. Barton. Modeling; simulation, sensitivity analysis and optimization hybrid systems. ACM Transactions on Modeling Computer Simulation, 2001. submitted.

[12] J. E. Tolsma and P. I. Barton. Computation of heteroazeotropes. I: Theory. Chemical Engineering Science, 55(18):3817-3834, 2000.

[13] J. E. Tolsma and P. I. Barton. Computation of heteroazeotropes. II: Efficient calculation of changes in phase equilibrium structure. Chemical Engineering Science, 55(18):3835-3853, 2000. 


\section{Subject Categories (keywords)}

Hybrid systems; dynamic optimization; sensitivity analysis; batch process design; azeotropic distillation.

\section{Description/Abstract}

Final technical report for project entitled "Synthesis and Optimization of Integrated Chemical Processes". Progress is reported on novel algorithms for the computation of all heteroazeotropic compositions present in complex liquid mixtures; the design of novel flexible azeotropic separation processes using middle vessel batch distillation columns; and theory and algorithms for sensitivity analysis and numerical optimization of hybrid discrete/continuous dynamic systems. 